\title{
Dietary Total Antioxidant Capacity and Gestational Diabetes Mellitus: A Case-Control Study
}

\author{
Elnaz Daneshzad, ${ }^{1}$ Hatav Tehrani, ${ }^{2}$ Nick Bellissimo, ${ }^{3}$ and Leila Azadbakht $\mathbb{D}^{1,4,5}$ \\ ${ }^{1}$ Department of Community Nutrition, School of Nutritional Science and Dietetics, Tehran University of Medical Sciences, \\ Tehran, Iran \\ ${ }^{2}$ Department of Obstetrics and Gynecology, Isfahan University of Medical Sciences, Isfahan, Iran \\ ${ }^{3}$ School of Nutrition, Ryerson University, Toronto, Canada \\ ${ }^{4}$ Diabetes Research Center, Endocrinology and Metabolism Clinical Sciences Institute, Tehran University of Medical Sciences, \\ Tehran, Iran \\ ${ }^{5}$ Department of Community Nutrition, School of Nutrition and Food Science, Isfahan University of Medical Science, Isfahan, Iran
}

Correspondence should be addressed to Leila Azadbakht; azadbakhtleila@gmail.com

Received 22 April 2020; Accepted 11 August 2020; Published 8 October 2020

Academic Editor: Manuela Curcio

Copyright (c) 2020 Elnaz Daneshzad et al. This is an open access article distributed under the Creative Commons Attribution License, which permits unrestricted use, distribution, and reproduction in any medium, provided the original work is properly cited.

\begin{abstract}
Background. Elevated oxidative stress status has been reported among pregnant women with gestational diabetes mellitus (GDM). In diabetic condition, glucose and lipid peroxidation, and alteration in antioxidant defense lead to increased free radicals. The objective of this study was to investigate the association between dietary total antioxidant capacity (DTAC) and GDM. Methods. This hospital-based case-control study was conducted in 463 pregnant women (healthy, $n=263$; GDM, $n=200$ ). Anthropometric indices, blood pressure, and biochemical analyses were measured. Dietary intake was assessed by the average of three 24-hour dietary intake records. DTAC was calculated by three indices: ferric reducing ability of plasma (FRAP), total radical-trapping antioxidant parameter (TRAP), and Trolox equivalent antioxidant capacity (TEAC). Multivariable logistic regression was performed to examine the relationship between DTAC and GDM risk in crude and adjusted models. Results. The mean age and BMI were $28.33 \pm 6.23$ years and $29.67 \pm 4.73 \mathrm{~kg} / \mathrm{m}^{2}$, respectively. Total energy, protein, and selenium intakes were significantly higher in cases than controls $(P<0.05)$. Moreover, intakes of carbohydrate, vitamins $\mathrm{C}, \mathrm{B} 6$, and $\mathrm{A}$, manganese, fruits, fruit juices, vegetables, legumes, and FRAP were significantly lower in cases than controls $(P<0.05)$. The risk of gestational diabetes mellitus was $85 \%$ lower among those in the highest tertile of FRAP (OR: 0.15; 95\% CI: 0.08-0.29). There was no significant association between the risk of GDM and TRAP (OR: 1.62; 95\% CI: 0.94-2.79) as well as TEAC (OR: 1.56; 95\% CI: 0.89-2.72). Conclusion. Pregnant women who were in the highest tertile of FRAP were at lower risk of GDM. However, larger prospective studies are needed to confirm our findings.
\end{abstract}

\section{Introduction}

Gestational diabetes mellitus (GDM) is a condition in which glucose metabolism is abnormal during the second and third trimester of pregnancy [1]. Early diagnosis and treatment are important because blood glucose disturbances might lead to fetal hypoxia, fetal malformation, and respiratory distress syndrome, to name a few [2-4]. Moreover, GDM may also be associated with developing type 2 diabetes, metabolic syndrome, and cardiovascular disease later in life [5]. Some studies have also reported the presence of severe oxidative stress status in diabetes as well as GDM [6, 7]. Glucose and lipid peroxidation, an alteration in antioxidant defense and oxidative destruction of glycated proteins, can lead to an increase in the production of free radicals [8-10]. Moreover, oxidative stress increases during pregnancy due to an increase in oxygen utilization and imbalance between production and scavenging of free radicals [11, 12]. An important role of dietary components is suggested as antioxidants can inhibit the activity of free radicals and neutralize them 
[12]. Healthy diets can improve abnormal glucose tolerance. Dietary patterns including fruits, low-fat dairies, and vegetables are associated with reduced risk of GDM [13]. In a previous study, higher intake of fruits and vegetables was associated with lower abnormal glucose tolerance in pregnant women [14]. For example, adherence to the Mediterranean and Dietary Approaches to Stop Hypertension (DASH) diet, which is rich in fruits and vegetables, is associated with reduced risk of GDM [15].

Diets rich in antioxidants improve total antioxidant status and lead to better health [16]. Measurement of total antioxidant capacity (TAC) is useful to determine the relationship to health, and the cumulative effect of antioxidants [17] may exert additive effects among antioxidants [18]. Dietary total antioxidant capacity (DTAC) shows the cumulative antioxidant capacity of foods and beverages, specifically fruits, fruits juices, and vegetables [19]. In a recent systematic review, an inverse association between DTAC and blood glucose concentrations was observed [20] through its effects on glucose metabolism [21]. Other studies have shown an inverse association between DTAC and risk of some chronic disorders such as cancer [22, 23], development of metabolic syndrome [24], lipid profile [25], inflammation [26], and biomarkers of diabetes [27].

To the best of our knowledge, there is no study which has assessed whole dietary antioxidant capacity and GDM in Iran. Therefore, this study is aimed at investigating the association between dietary total antioxidant capacity and GDM in a case-control study.

\section{Methods}

The present study was as a hospital-based case-control study, conducted in a referral hospital and nutrition clinics in Isfahan, Iran. Pregnant women ( $n=463$; based on simple sample collection and using following formula: $(r+1 / r)$ $\left(\mathrm{SD}^{2}\left(Z_{\beta}+Z_{\alpha} / 2\right)^{2} / d^{2}\right.$; using fasting blood sugar variable $=$ $109.2 ; r=1, Z_{\beta}=0.84$, and $Z_{\alpha / 2}=1.96$; power of $80 \%$ ) [28] with a singleton pregnancy between the ages of 22 to $44 \mathrm{y}$ participated in this study. Participants were between 25 and 28 weeks of pregnancy. In this study, 200 subjects had GDM and 263 women did not have GDM. Cases were chosen from Azzahra hospital, nutrition clinics, and Shahid Beheshti hospital, and controls were chosen from outpatients who referred to Azzahra and Shahid Beheshti hospital in Isfahan. Controls were the patients who attend a hospital for treatment without staying there overnight. Informed written consent was obtained from all participants. For GDM cases, we included pregnant women with abnormal fasting glucose (FG $\geq 95 \mathrm{mg} / \mathrm{dl}$ or 1-hour postprandial glucose $\geq 140 \mathrm{mg} / \mathrm{dl}$ for the first screening in pregnancy duration) [29]. Moreover, GDM was diagnosed by medical doctors. GDM was diagnosed between the 25th and 28th week of pregnancy. The participants were the new diagnosed pregnant which did not use any insulin or medication therapy. Moreover, the controls were non-GDM pregnant who their FG or 1-hour postprandial glucose levels were normal. Every pregnant participant with GDM was matched with a non-GDM pregnant for the specific week of gestational period (between 25 and 28 weeks of pregnancy). Women who were carrying multiple fetuses or women with diabetes, cancer, cardiovascular diseases and who reported any medication or hormone therapy and incomplete daily food records or abnormal energy intake $(<800$ or $>4200 \mathrm{kcal} / \mathrm{d})$ were excluded. This study has been approved by Isfahan University of Medical Sciences, Isfahan, Iran.

Participant weight was measured to the nearest $100 \mathrm{~g}$ on a SECA scale without shoes and wearing light clothing. Height was measured using a wall tape while barefoot. Body mass index (BMI) was calculated by weight $(\mathrm{kg}) /$ height $\left(\mathrm{m}^{2}\right)$. Blood pressure was measured twice after 5 min of rest using an appropriate cuff according to arm size. The average of two blood pressure measurements was recorded as the final value. Blood samples were obtained after 12-hour overnight fast and were centrifuged within 30-45 minutes of collection for 10 minutes at $500 \times \mathrm{g}$ and at $4^{\circ} \mathrm{C}$. Blood samples were analyzed using an autoanalyzer (Selectra 2; Vital Scientific, Spankeren, Netherlands). Triacylglycerols (TGs) were measured with glutathione peroxidase. Aspartate aminotransferase (AST), alanine aminotransferase (ALT), low-density lipoprotein cholesterol (LDL-C), high-density lipoprotein cholesterol (HDL-C), serum total cholesterol (TC), and FG were measured using commercially available enzymatic kits (Pars Azmmoun, Tehran, Iran).

Dietary intake was assessed by the average of three 24-hour dietary records ( 2 weekday and 1 weekend). Participants were trained by an expert dietitian to complete the dietary food record forms. Food album was used to estimate and record the correct size of food portions. Moreover, the raw and cooked coefficients were considered for all foods. The dietitian checked the forms to ensure their accuracy and followed up with participants by phone when records were incomplete. All portion sizes of food records were converted to grams using household measures of Iranian foods. The Nutritionist IV software (First Databank Division, the Hearst Corporation, San Bruno, CA, USA), modified for Iranian foods, was used to assess the dietary intake.

The quantitative value of DTAC for dietary intake was calculated by three indices: ferric reducing ability of plasma (FRAP), total radical-trapping antioxidant parameter (TRAP), and Trolox equivalent antioxidant capacity (TEAC). FRAP value was obtained using published databases developed by Halvorsen et al. [30]. TRAP and TEAC values were obtained by using previously published databases for Italian foods [31]. TAC value for food items was matched to an equivalent food in each of the databases. If each food was not directly matched in a database, a proxy estimation was used based on the following procedure: (1) the mean value of similar foods when no data were available and (2) TAC values of ingredients based on portions of processed or dish-based foods.

Dietary total antioxidant capacity for every participant was obtained by multiplying the daily intake of each selected food item by its corresponding antioxidant value per food portion and summing the final values. Antioxidants from supplements were not considered in the calculation of DTAC. 
Socioeconomic information of participants was obtained by health care providers. Socioeconomic status (SES) was assessed by asking the pregnant women about their education status (academic or nonacademic), car ownership, house ownership (yes and no), and family size ( $\leq 4$ and $>4$ persons). A score of 0.5 was given for having family members of $\leq 4$, academic educations, and house or car ownership to each subject. If they had family members of $>4$, had nonacademic educations, or were not home or car owners, they were given the score of 0 . Finally, the scores were summed and presented as total SES score. Physical activity was recorded in MET/d by participants. Participants were asked about smoking habits; however, all of them were nonsmokers. Moreover, participants were asked about their supplementation intakes. All participants consumed iron supplement.

Energy intake, sociodemographic, anthropometric, and biochemical indices were compared among case and control groups using the independent $t$-test for quantitative variables and chi-square test for the categorical variables. Analysis of covariance (ANCOVA) was used to assess the dietary intake of case and control group by adjusting energy intake. Multivariate logistic regression was performed to examine the relationship between DTAC and GDM risk in crude and adjusted models. A crude model was performed without adjustment, and two adjusted models were performed to control the effects of potential confounders (model 1: age and energy intake, model 2: model 1+socioeconomic status; SES score, model 3: models 1 and 2+dietary fiber intake, model 4: models 1, 2, and 3+carbohydrate and protein intake; and model 5: further adjustment for BMI, supplementation, physical activity, and fat intake). Statistical analyses were performed using the SPSS software (SPSS Inc., Chicago IL, version 16), and $P<0.05$ was considered as statistically significant.

\section{Results}

The mean age and BMI were $28.33 \pm 6.23$ years and 29.67 $\pm 4.7 \mathrm{~kg} / \mathrm{m}^{2}$, respectively. Table 1 shows the anthropometric and biochemical indices in the case (GDM) and control (healthy) groups. The mean FG, HbAlC, and TG were higher in cases compared to controls $(P<0.0001)$.

Figure 1 is a comparison of lipid profile and liver enzymes in GDM and healthy pregnant women across tertiles of DTAC. In Figure 1(a), TG levels were significantly lower in the highest tertile of FRAP value in healthy individuals (tertile 1: $144.02 \pm 6.04$; tertile 2: $129.21 \pm 4.25$; tertile 3: $121.24 \pm 4.09)$; however, other variables did not indicate a statistical association. TG levels were significantly lower in the highest tertile of TRAP value in healthy individuals (tertile 1: $127.01 \pm 4.16$; tertile 2: $138.09 \pm 4.55$; tertile 3: $122.78 \pm 4.51 ; P=0.04)$.

Dietary intake and dietary total antioxidant capacity among the case and control groups are indicated in Table 2. Total energy, protein, and selenium intakes were significantly higher in cases than controls $(P<0.05)$. Moreover, intakes of carbohydrate, vitamins $\mathrm{C}, \mathrm{B} 6$, and A, manganese, fruits, fruit juices, vegetables, legumes, and FRAP value were significantly lower in cases than controls $(P<0.05)$. There was no significant difference for TRAP and TEAC values between cases and controls $(P>0.05)$.

Multivariate odds ratios for GDM across tertiles of DTAC are reported in Table 3 in crude and adjusted models. The risk of gestational diabetes mellitus was $85 \%$ lower among those in the highest tertile of FRAP. There was no significant association between TEAC and TRAP with the risk of GDM.

\section{Discussion}

In this case-control study, mean FRAP and vitamin $\mathrm{C}$ were lower in GDM cases compared with a healthy control group. Based on these results, dietary total antioxidant capacity may reduce abnormal blood glucose level in pregnant women. Although there was no significant association between GDM and tertiles of TRAP and TEAC, there was a significant inverse association between GDM and FRAP. To the best of our knowledge, this is the first study that has investigated the association between GDM and DTAC.

According to several studies in healthy adults, adjusted DTAC had an inverse association with fasting blood glucose and insulin levels $[32,33]$. In previous studies, the food frequency questionnaire was used to assess dietary intake. In addition, age, gender, and energy intake were considered as potential confounders [24, 32-34]. In agreement with our study, a case-control study of 80 diabetic patients and 37 controls found lower DTAC status in diabetic patients [35].

Consistent with our study, another case-control study found that prediabetes individuals have significantly lower mean DTAC than the control group [28]. Participants who were in the highest quartile of DTAC were less likely to experience elevated blood glucose concentrations after, adjusting BMI, dietary fiber, fat, and energy intake [28]. A crosssectional study has also founded that higher DTAC is associated with lower levels of diabetes biomarkers in healthy prediabetic and diabetic individuals [27]. In our study, the significant association between DTAC and GDM persisted on even after adjusting potential confounders such as age and energy intake.

Fruits are rich source of antioxidants as well as vitamins, minerals, terpenes, lignans, polyphenols, and flavonoids. Positive associations have been observed between plasma TAC and fruit, moreover between plasma TAC and vegetable intake [36]. Fruits have low energy density and low glycemic load; moreover, their antioxidant capacity leads to a diminishment of oxidative stress, which is why they are beneficial for increasing insulin sensitivity and pancreatic $\beta$ cell function [37-39]. Whole grains have high antioxidant capacity, in addition, contribute to lower absorption of glucose [40]. Fruits, vegetables, and whole grains as sources of dietary fiber have beneficial effects on diabetes-related biomarkers [41]. In the present study, the association between FRAP value and GDM remained significant even after adjusting dietary fiber.

Pancreatic $\beta$ cells are vulnerable to the effects of oxidative stress due to lack of enzymatic antioxidants; therefore, oxidative stress, which damages mitochondria, reduces insulin secretion and increases blood glucose $[42,43]$. A decline in 
TABLe 1: Anthropometric and biochemical indices among the cases* and control groups.

\begin{tabular}{|c|c|c|c|}
\hline \multirow{2}{*}{ Variables } & \multicolumn{2}{|c|}{ Groups, mean \pm SD } & \multirow{2}{*}{$P$ value } \\
\hline & Case $(n=200)$ & Control $(n=263)$ & \\
\hline Age, y & $29.27 \pm 5.96$ & $27.61 \pm 6.34$ & 0.004 \\
\hline Weight, kg & $74.45 \pm 13.10$ & $74.53 \pm 12.97$ & 0.943 \\
\hline BMI, kg/m² & $29.67 \pm 4.88$ & $29.67 \pm 4.62$ & 0.994 \\
\hline Waist circumference, $\mathrm{cm}$ & $96.17 \pm 15.03$ & $98.11 \pm 17.73$ & 0.215 \\
\hline Physical activity, MET/h & $9.61 \pm 0.48$ & $9.59 \pm 0.46$ & 0.749 \\
\hline $\mathrm{SBP}, \mathrm{mmHg}$ & $120.15 \pm 15.61$ & $120.26 \pm 17.72$ & 0.464 \\
\hline $\mathrm{DBP}, \mathrm{mmHg}$ & $74.92 \pm 9.01$ & $76.11 \pm 8.50$ & 0.130 \\
\hline $\mathrm{FG}, \mathrm{mg} / \mathrm{dl}$ & $148.00 \pm 48.92$ & $78.20 \pm 10.94$ & $<0.0001$ \\
\hline HbA1C (\%) & $7.81 \pm 1.68$ & $5.58 \pm 1.16$ & $<0.0001$ \\
\hline TC, mg/dl & $179.07 \pm 62.38$ & $170.90 \pm 38.14$ & 0.083 \\
\hline $\mathrm{TG}, \mathrm{mg} / \mathrm{dl}$ & $172.14 \pm 86.32$ & $128.87 \pm 40.69$ & $<0.0001$ \\
\hline LDL-C, mg/dl & $95.52 \pm 30.03$ & $93.53 \pm 34.33$ & 0.516 \\
\hline HDL-C, mg/dl & $45.60 \pm 9.43$ & $47.25 \pm 9.17$ & 0.059 \\
\hline ALT, IU/L & $21.99 \pm 14.74$ & $20.11 \pm 10.57$ & 0.112 \\
\hline AST, IU/L & $22.57 \pm 15.62$ & $22.60 \pm 12.98$ & 0.977 \\
\hline SES score & $1.63 \pm 0.90$ & $1.45 \pm 0.80$ & 0.027 \\
\hline Multivitamin intake; $n(\%)$ & $39(19.5)$ & $50(19.2)$ & 0.895 \\
\hline
\end{tabular}

${ }^{*}$ Case: women with gestational diabetes mellitus. BMI: body mass index; SBP: systolic blood pressure; DBP: diastolic blood pressure; FG: fasting glucose; HbA1c: hemoglobin A1C; TC: total cholesterol; TG: triglyceride; LDL-C: low-density lipoprotein cholesterol; HDL-C: high-density lipoprotein cholesterol; ALT: alanine aminotransferase; AST: aspartate aminotransferase; SES: socioeconomic status.

antioxidant defense and increase in radical oxygen species can lead to damage of cellular organelles and enzymes, increase proteins and lipids peroxidation, and develop insulin resistance $[44,45]$. Therefore, the adequate intake of antioxidants may be important for the maintenance of glucose homeostasis.

Abnormal glucose metabolism and increased glycemia condition can develop complications and health disturbances which lead to fetal hypoxia, fetal malformation, and respiratory distress syndrome; therefore, early diagnosis and all kind of treatment approaches such as improving lifestyle, diet therapy, and healthy food choices are important to control the blood glucose levels during pregnancy and reduce the probability of developing type 2 diabetes, metabolic syndrome, and cardiovascular disease later in life [2-4]. Increased weight during pregnancy develops insulin resistance. Moreover, insulin resistance and obesity increase oxidative stress in women with GDM. Therefore, consuming high antioxidant foods or nutrients and increased dietary antioxidant capacity may contribute to delay or prevent the initiation or progression of GDM [3-5].

In the present study, TG levels were significantly lower in the highest tertile of FRAP value in healthy individuals. Also, TG levels were significantly lower in the highest tertile of TRAP value in healthy individuals. Inconsistent results are presented in different studies. Bahadoran et al. and Georgoulis et al. [46, 47] failed to find any significant association between DTAC and TG. In line with our study, a study showed an inverse association between DTAC and TG; how- ever, DTAC was measured by TEAC [24]. Moreover, Kim et al. revealed an inverse association between DTAC quartiles and TG [25]. Dietary antioxidants contribute to reducing cholesterol absorption and suppressing cholesterol synthesis and intermediate role of insulin resistance [48].

In the result description, we should consider that DTAC was based on dietary antioxidants and did not consider antioxidant bioavailability or metabolism. Moreover, three issues for assessing DTAC are developed as rapid in vitro methods and are different in basic calculation. In fact, these three methods differ on the chemical background and nature of redox system. TRAP measures the chain-breaking antioxidant capacity and involves the transfer of hydrogen atoms; FRAP measures the iron-reducing power of antioxidants, and TEAC measures the antioxidant capacity to quench free radicals and involve electron transfer reactions. These three databases include different numbers of foods such as vegetables, fruits, nuts, and dried fruits.

The present study has several limitations. The casecontrol design is the main limitation due to its inability to support cause and effect relationships. Also, one of the well-known limitations is the use of a 24-hour dietary record to assess dietary intake which leads to reproducibility and recall bias. Moreover, the findings of this study might not be generalizable to other populations without GDM. It might not be useful for FRAP assay for assessing total antioxidant capacity levels and its association with GDM. As there is no database for the content of Iranian food antioxidant, using a database for Italian food is another limitation of the study. 


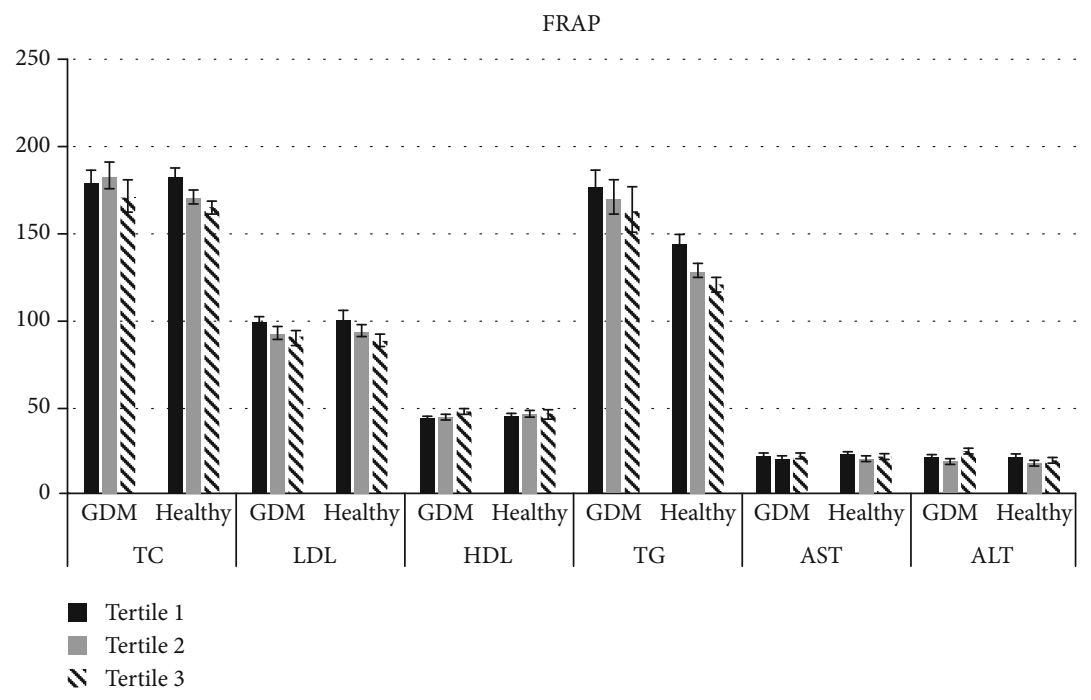

(a)

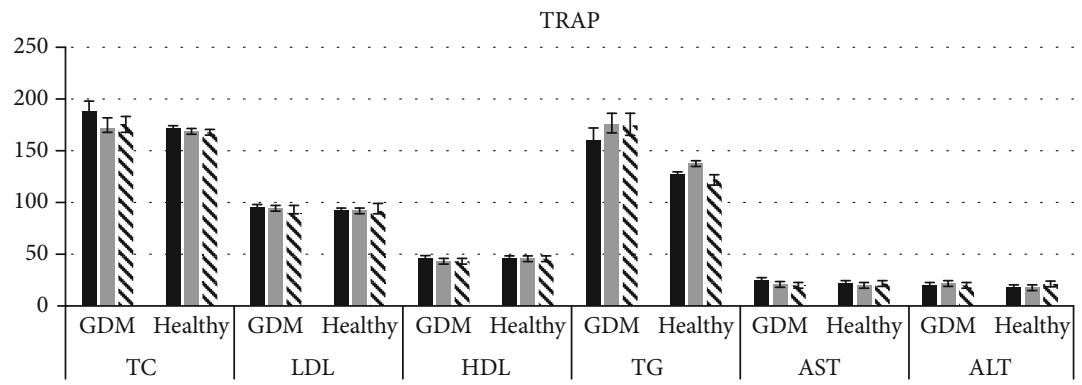

a Tertile

Tertile 2

N Tertile 3

(b)

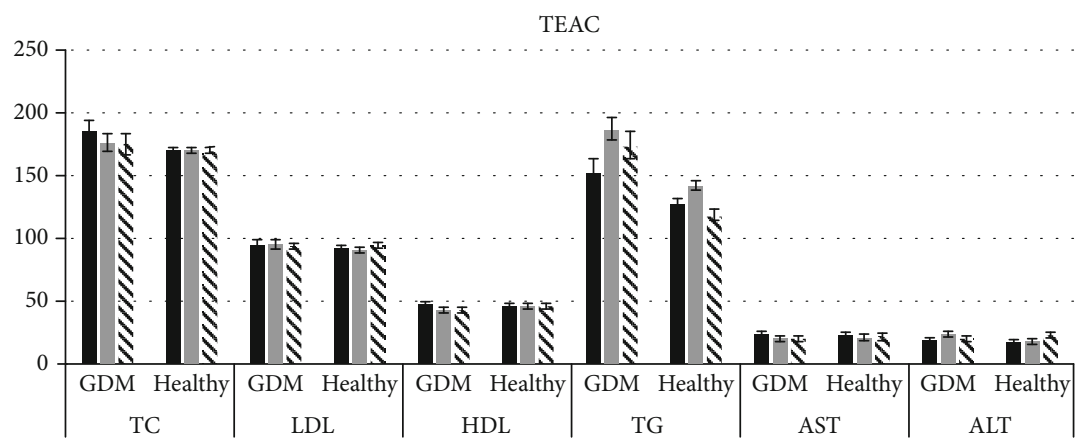

- Tertile 1

- Tertile 2

N Tertile 3

(c)

FIgURe 1: Comparison of lipid profile (mg/dl) and liver enzymes (IU/L) in GDM and healthy pregnant women among different tertiles of DTAC (presented as mean and standard error in three parts: $(a-c)$ ). (a) Comparison of lipid profile and liver enzymes in cases and controls among different tertiles of FRAP using ANCOVA. Variables have adjusted for age, energy intake, SES, number of having child, and dietary fiber intake. TG in healthy group is significant $(P<0.05)$. (b) Comparison of lipid profile and liver enzymes in cases and controls among different tertiles of TRAP using ANCOVA. Variables have adjusted for age, energy intake, SES, number of having child, and dietary fiber intake. TG and ALT in healthy group are significant $(P<0.05)$. (c) Comparison of lipid profile and liver enzymes in cases and controls among different tertiles of TEAC using ANCOVA. Variables have adjusted for age, energy intake, SES, number of having child, and dietary fiber intake. TG and ALT in healthy group and HDL in GDM group are significant $(P<0.05)$. 
TABLE 2: Dietary intake and dietary total antioxidant capacity among the cases* and control groups.

\begin{tabular}{|c|c|c|c|}
\hline \multirow{2}{*}{ Dietary intake } & \multicolumn{2}{|c|}{ Groups } & \multirow{2}{*}{$P$ value $^{\infty}$} \\
\hline & Case $(n=200)$ & Control $(n=263)$ & \\
\hline Total energy intake, kcal & $1970.32 \pm 500.06$ & $1861.89 \pm 612.86$ & 0.042 \\
\hline Carbohydrate, g & $320.87 \pm 2.79$ & $329.09 \pm 2.43$ & 0.027 \\
\hline Fat, g & $46.27 \pm 1.19$ & $45.03 \pm 1.04$ & 0.433 \\
\hline Protein, $g$ & $67.57 \pm 1.16$ & $62.71 \pm 1.01$ & 0.002 \\
\hline Fiber, g & $26.51 \pm 0.58$ & $26.60 \pm 0.50$ & 0.906 \\
\hline Vitamin $\mathrm{D}, \mu \mathrm{g} / \mathrm{d}$ & $1.30 \pm 0.09$ & $1.17 \pm 0.07$ & 0.308 \\
\hline Vitamin $\mathrm{C}, \mathrm{mg} / \mathrm{d}$ & $265.18 \pm 6.55$ & $307.41 \pm 5.72$ & $<0.0001$ \\
\hline Vitamin E, IU/d & $7.30 \pm 0.35$ & $7.35 \pm 0.31$ & 0.913 \\
\hline Vitamin A, IU/d & $1385.87 \pm 58.80$ & $1572.87 \pm 51.35$ & 0.017 \\
\hline Calcium, mg/d & $1248.56 \pm 31.74$ & $1270.27 \pm 27.71$ & 0.607 \\
\hline Zinc, mg/d & $8.89 \pm 0.14$ & $9.13 \pm 0.13$ & 0.228 \\
\hline Magnesium, mg/d & $369.21 \pm 5.97$ & $371.50 \pm 5.21$ & 0.773 \\
\hline Vitamin $\mathrm{B}_{2}, \mathrm{IU} / \mathrm{d}$ & $2.25 \pm 0.04$ & $2.31 \pm 0.03$ & 0.227 \\
\hline Vitamin $\mathrm{B}_{6}, \mathrm{mg} / \mathrm{d}$ & $1.71 \pm 0.03$ & $1.82 \pm 0.02$ & 0.008 \\
\hline Selenium, $\mu \mathrm{g} / \mathrm{d}$ & $0.07 \pm 0.002$ & $0.06 \pm 0.002$ & 0.002 \\
\hline Iron, mg/d & $12.51 \pm 0.19$ & $12.53 \pm 0.17$ & 0.919 \\
\hline Manganese, mg/d & $4.12 \pm 0.10$ & $4.41 \pm 0.08$ & 0.028 \\
\hline Fruits, g & $584.36 \pm 15.84$ & $663.781 \pm 13.91$ & $<0.0001$ \\
\hline Fruit juices, $g$ & $5.44 \pm 1.10$ & $8.50 \pm 0.96$ & 0.038 \\
\hline Vegetables, g & $449.54 \pm 15.64$ & $500.39 \pm 13.73$ & 0.015 \\
\hline Nuts, g & $11.45 \pm 1.29$ & $14.50 \pm 1.13$ & 0.077 \\
\hline Egg, g & $9.10 \pm 0.56$ & $10.26 \pm 0.72$ & 0.125 \\
\hline Rice, $g$ & $317.27 \pm 12.05$ & $328.95 \pm 10.52$ & 0.467 \\
\hline Legumes, g & $22.14 \pm 1.23$ & $28.73 \pm 1.08$ & $<0.0001$ \\
\hline Grains, g & $402.83 \pm 11.93$ & $402.90 \pm 10.41$ & 0.997 \\
\hline Red meat, g & $8.12 \pm 0.37$ & $8.57 \pm 0.32$ & 0.361 \\
\hline Fish and chicken, $g$ & $14.73 \pm 0.74$ & $17.51 \pm 0.64$ & 0.005 \\
\hline Processed meat, g & $1.78 \pm 0.34$ & $5.18 \pm 0.30$ & $<0.0001$ \\
\hline TRAP, mmol/d & $8.34 \pm 0.26$ & $8.60 \pm 0.23$ & 0.462 \\
\hline FRAP, $\mathrm{mmol} / \mathrm{d}$ & $11.13 \pm 0.28$ & $12.88 \pm 0.25$ & $<0.0001$ \\
\hline TEAC, $\mathrm{mmol} / \mathrm{d}$ & $7.18 \pm 0.20$ & $7.45 \pm 0.17$ & 0.311 \\
\hline
\end{tabular}

${ }^{*}$ Case: women with gestational diabetes mellitus. ${ }^{\infty}$ All food values presented as mean and standard error using ANCOVA test which adjusted for energy intake. Total energy intake presented as mean and standard deviation ANOVA. TRAP: total radical-trapping antioxidant parameter; FRAP: ferric reducing ability of plasma; TEAC: Trolox equivalent antioxidant capacity.

Finally, although we assessed the supplement intake and adjusted their residual effect in the analysis, we just calculated the dietary antioxidant capacity and did not consider the supplement intake for calculation of antioxidant capacity. On the other hand, the strengths of the present study are adequate sample size and adjustment for several potential confounders. Additionally, despite there is no valuable data about DTAC of Iranian foods, we included all three assays for calculating DTAC.

The present study is aimed at investigating the association between dietary total antioxidant capacity and GDM.
Based on these results, pregnant women who were in the highest tertile of FRAP were at lower risk for GDM. As it is well-known that healthy eating patterns have important applications to the prevention and treatment of chronic diseases, dietary antioxidant consumption may be have beneficial effects in this regard and may be related to clinical practices. Future clinical trials that consider various confounders and larger prospective studies with other dietary assessment tools such as dietary history questionnaires or food frequency questionnaires are needed to find the exact relationships. 
TABLE 3: Odd ratio for gestational diabetes mellitus in different tertiles of dietary total antioxidant capacity indices.

\begin{tabular}{|c|c|c|c|c|}
\hline & 1 & 2 & 3 & $P$ trend $^{\infty}$ \\
\hline \multicolumn{5}{|c|}{ Tertiles of TRAP } \\
\hline Crude model & 1 & $1.17(1.08-2.69)$ & $1.38(0.87-2.19)$ & 0.167 \\
\hline Model 1 & 1 & $1.60(0.99-2.56)$ & $1.25(0.76-2.06)$ & 0.381 \\
\hline Model 2 & 1 & $1.63(1.00-2.63)$ & $1.38(0.83-2.30)$ & 0.211 \\
\hline Model 3 & 1 & $1.61(0.99-2.61)$ & $1.36(0.81-2.27)$ & 0.237 \\
\hline Model 4 & 1 & $1.53(0.94-2.51)$ & $1.45(0.85-2.45)$ & 0.156 \\
\hline Model 5 & 1 & $1.49(0.91-2.45)$ & $1.62(0.94-2.79)$ & 0.072 \\
\hline \multicolumn{5}{|c|}{ Tertiles of FRAP } \\
\hline Crude model & 1 & $0.44(0.27-0.70)$ & $0.26(0.16-0.42)$ & $<0.0001$ \\
\hline Model 1 & 1 & $0.40(0.25-0.66)$ & $0.23(0.13-0.38)$ & $<0.0001$ \\
\hline Model 2 & 1 & $0.43(0.26-0.71)$ & $0.23(0.14-0.40)$ & $<0.0001$ \\
\hline Model 3 & 1 & $0.36(0.21-0.61)$ & $0.16(0.09-0.30)$ & $<0.0001$ \\
\hline Model 4 & 1 & $0.33(0.19-0.57)$ & $0.15(0.08-0.29)$ & $<0.0001$ \\
\hline Model 5 & 1 & $0.31(0.17-0.53)$ & $0.15(0.08-0.29)$ & $<0.0001$ \\
\hline \multicolumn{5}{|c|}{ Tertiles of TEAC } \\
\hline Crude model & 1 & $1.50(0.95-2.37)$ & $1.34(0.85-2.10)$ & 0.201 \\
\hline Model 1 & 1 & $1.35(0.84-2.18)$ & $1.18(0.72-1.95)$ & 0.509 \\
\hline Model 2 & 1 & $1.52(0.93-2.48)$ & $1.40(0.83-2.36)$ & 0.204 \\
\hline Model 3 & 1 & $1.52(0.93-2.48)$ & $1.36(0.80-2.31)$ & 0.243 \\
\hline Model 4 & 1 & $1.37(0.83-2.26)$ & $1.37(0.80-2.34)$ & 0.239 \\
\hline Model 5 & 1 & $1.32(0.80-2.19)$ & $1.56(0.89-2.72)$ & 0.113 \\
\hline
\end{tabular}

${ }^{\infty} P$ trends calculated by binary logistic regression. Model 1 has adjusted for age and energy intake. Model 2 is model 1+SES and number of offspring. Model 3 is models 1 and 2+dietary fiber intake. Model 4 is models 1, 2, and $3+$ carbohydrate and protein intake. Model 5 is the further adjustment for BMI, supplementation, physical activity, and fat intake. TRAP: total radical-trapping antioxidant parameter; FRAP: ferric reducing ability of plasma; TEAC: Trolox equivalent antioxidant capacity.

\section{Data Availability}

The data used to support the findings of this study are available from the corresponding author upon request.

\section{Ethical Approval}

The present study was approved at Tehran University of Medical Sciences.

\section{Disclosure}

The abstract of the present study was presented as a poster in the Third International Congress and Fifteenth Iranian Nutrition Congress.

\section{Conflicts of Interest}

The authors declare no conflict of interest.

\section{References}

[1] American Diabetes Association, "2. Classification and diagnosis of diabetes," Diabetes Care, vol. 39, Supplement 1, pp. S13-S22, 2016.
[2] Q. Zhang, Y. Cheng, M. He, T. Li, Z. Ma, and H. Cheng, "Effect of various doses of vitamin D supplementation on pregnant women with gestational diabetes mellitus: a randomized controlled trial," Experimental and Therapeutic Medicine, vol. 12, no. 3, pp. 1889-1895, 2016.

[3] M. Aittasalo, J. Raitanen, T. I. Kinnunen, K. Ojala, P. Kolu, and R. Luoto, "Is intensive counseling in maternity care feasible and effective in promoting physical activity among women at risk for gestational diabetes? Secondary analysis of a cluster randomized NELLI study in Finland," The International Journal of Behavioral Nutrition and Physical Activity., vol. 9, no. 1, p. 104, 2012.

[4] G. Xin, J. Du, Y. T. Wang, and T. T. Liang, "Effect of oxidative stress on heme oxygenase-1 expression in patients with gestational diabetes mellitus," Experimental and Therapeutic Medicine, vol. 7, no. 2, pp. 478-482, 2014.

[5] E. Noctor, C. Crowe, L. A. Carmody et al., "ATLANTIC-DIP: prevalence of metabolic syndrome and insulin resistance in women with previous gestational diabetes mellitus by International Association of Diabetes in Pregnancy Study Groups criteria," Acta Diabetologica, vol. 52, no. 1, pp. 153-160, 2015.

[6] H. Z. Pan, L. Zhang, M. Y. Guo et al., "The oxidative stress status in diabetes mellitus and diabetic nephropathy," Acta Diabetologica, vol. 47, Supplement 1, pp. 71-76, 2010.

[7] C. M. John, R. Ramasamy, G. Al Naqeeb, A. H. Dhiab AlNuaimi, and A. Adam, "Enhanced CD4+CD25+ regulatory T cells with splenic proliferation and protection against oxidative stress by nicotinamide in gestational diabetes," Current Medicinal Chemistry, vol. 16, 2012.

[8] N. Altan, A. S. Dinçel, and C. Koca, "Diabetes mellitus ve oksidatif stres," Türk Biyokimya Dergisi, vol. 1, pp. 51-56, 2006.

[9] A. C. Maritim, R. A. Sanders, and J. B. Watkins 3rd., "Diabetes, oxidative stress, and antioxidants: a review," Journal of Biochemical and Molecular Toxicology, vol. 17, no. 1, pp. 24-38, 2003.

[10] G. Paolisso, A. D'Amore, G. di Maro et al., "Evidence for a relationship between free radicals and insulin action in the elderly," Metabolism: Clinical and Experimental, vol. 42, no. 5, pp. 659-663, 1993.

[11] M. M. Al-Shebly and M. A. Mansour, "Evaluation of oxidative stress and antioxidant status in diabetic and hypertensive women during labor," Oxidative Medicine and Cellular Longevity, vol. 2012, Article ID 329743, 6 pages, 2012.

[12] E. Granot and R. Kohen, "Oxidative stress in childhood-in health and disease states," Clinical Nutrition, vol. 23, no. 1, pp. 3-11, 2004.

[13] S. Zareei, R. Homayounfar, M. . Naghizadeh, E. Ehrampoush, and M. Rahimi, "Dietary pattern in pregnancy and risk of gestational diabetes mellitus (GDM)," Diabetes \& Metabolic Syndrome, vol. 12, no. 3, pp. 399-404, 2018.

[14] R. Mercier, J. Perron, S. J. Weisnagel, and J. Robitaille, “Associations between fruit and vegetables intake and abnormal glucose tolerance among women with prior gestational diabetes mellitus," European Journal of Nutrition, vol. 58, no. 2, pp. 689-696, 2019.

[15] V. Izadi, H. Tehrani, F. Haghighatdoost, A. Dehghan, P. J. Surkan, and L. Azadbakht, "Adherence to the DASH and Mediterranean diets is associated with decreased risk for gestational diabetes mellitus," Nutrition, vol. 32, no. 10, pp. 1092-1096, 2016. 
[16] E. Limberaki, P. Eleftheriou, E. Vagdatli, V. Kostoglou, and C. Petrou, "Serum antioxidant status among young, middleaged and elderly people before and after antioxidant rich diet," Hippokratia, vol. 16, no. 2, pp. 118-123, 2012.

[17] O. Erel, "A novel automated direct measurement method for total antioxidant capacity using a new generation, more stable ABTS radical cation," Clinical Biochemistry, vol. 37, no. 4, pp. 277-285, 2004.

[18] S. M. Hosseini-Zijoud, S. A. Ebadi, M. T. Goodarzi et al., "Lipid peroxidation and antioxidant status in patients with medullary thyroid carcinoma: a case-control study," Journal of Clinical and Diagnostic Research, vol. 10, no. 2, pp. Bc04-Bc07, 2016.

[19] M. Serafini and D. Del Rio, "Understanding the association between dietary antioxidants, redox status and disease: is the total antioxidant capacity the right tool?," Redox Report, vol. 9, no. 3, pp. 145-152, 2013.

[20] H. Mozaffari, E. Daneshzad, P. J. Surkan, and L. Azadbakht, "Dietary total antioxidant capacity and cardiovascular disease risk factors: a systematic review of observational studies," Journal of the American College of Nutrition, vol. 37, no. 6, pp. 533545, 2018.

[21] M. Sato, T. Miyazaki, T. Nagaya et al., “Antioxidants inhibit tumor necrosis factor-alpha mediated stimulation of interleukin-8, monocyte chemoattractant protein-1, and collagenase expression in cultured human synovial cells," The Journal of Rheumatology, vol. 23, no. 3, pp. 432-438, 1996.

[22] D. Gifkins, S. H. Olson, L. Paddock et al., "Total and individual antioxidant intake and risk of epithelial ovarian cancer," $B M C$ Cancer, vol. 12, no. 1, p. 211, 2012.

[23] C. la Vecchia, A. Decarli, M. Serafini et al., "Dietary total antioxidant capacity and colorectal cancer: a large case-control study in Italy," International Journal of Cancer, vol. 133, no. 6, pp. 1447-1451, 2013.

[24] H. H. M. Hermsdorff, B. Puchau, A. C. P. Volp et al., "Dietary total antioxidant capacity is inversely related to central adiposity as well as to metabolic and oxidative stress markers in healthy young adults," Nutrition \& Metabolism, vol. 8, no. 1, p. 59, 2011.

[25] K. Kim, T. M. Vance, and O. K. Chun, "Greater total antioxidant capacity from diet and supplements is associated with a Less atherogenic blood profile in U.S. adults," Nutrients, vol. 8, no. 1, p. 15, 2016.

[26] F. Brighenti, S. Valtueña, N. Pellegrini et al., "Total antioxidant capacity of the diet is inversely and independently related to plasma concentration of high-sensitivity C-reactive protein in adult Italian subjects," The British journal of Nutrition, vol. 93, no. 5, pp. 619-625, 2005.

[27] T. Psaltopoulou, D. B. Panagiotakos, C. Pitsavos et al., "Dietary antioxidant capacity is inversely associated with diabetes biomarkers: the ATTICA study," Nutrition, Metabolism, and Cardiovascular Diseases, vol. 21, no. 8, pp. 561-567, 2011.

[28] G. Sotoudeh, M. Abshirini, F. Bagheri, F. Siassi, F. Koohdani, and Z. Aslany, "Higher dietary total antioxidant capacity is inversely related to prediabetes: a case-control study," Nutrition, vol. 46, pp. 20-25, 2018.

[29] American Diabetes Association, "Standards of medical care in diabetes-2010," Diabetes Care, vol. 33, Supplement 1, pp. S11S61, 2009.

[30] B. L. Halvorsen, K. Holte, M. C. W. Myhrstad et al., "A systematic screening of total antioxidants in dietary plants," The Journal of Nutrition, vol. 132, no. 3, pp. 461-471, 2002.
[31] N. Pellegrini, M. Serafini, B. Colombi et al., "Total antioxidant capacity of plant foods, beverages and oils consumed in Italy assessed by three different in vitro assays," The Journal of Nutrition, vol. 133, no. 9, pp. 2812-2819, 2003.

[32] B. Puchau, M. Á. Zulet, A. G. de Echávarri, H. H. M. Hermsdorff, and J. A. Martínez, "Dietary total antioxidant capacity: a novel indicator of diet quality in healthy young adults," Journal of the American College of Nutrition, vol. 28, no. 6, pp. 648656, 2009.

[33] B. Puchau, M. A. Zulet, A. G. de Echávarri, H. H. M. Hermsdorff, and J. A. Martínez, "Dietary total antioxidant capacity is negatively associated with some metabolic syndrome features in healthy young adults," Nutrition, vol. 26, no. 5, pp. 534-541, 2010.

[34] H. Okubo, H. E. Syddall, D. I. Phillips et al., "Dietary total antioxidant capacity is related to glucose tolerance in older people: the Hertfordshire Cohort Study," Nutrition, Metabolism, and Cardiovascular Diseases, vol. 24, no. 3, pp. 301-308, 2014.

[35] M. E. Zujko, A. M. Witkowska, M. Gorska, J. Wilk, and A. Kretowski, "Reduced intake of dietary antioxidants can impair antioxidant status in type 2 diabetes patients," Polskie Archiwum Medycyny Wewnetrznej, vol. 124, no. 11, pp. 599607, 2014.

[36] C. Pitsavos, D. B. Panagiotakos, N. Tzima et al., "Adherence to the Mediterranean diet is associated with total antioxidant capacity in healthy adults: the ATTICA study," The American Journal of Clinical Nutrition, vol. 82, no. 3, pp. 694-699, 2005.

[37] A. Ceriello, "Oxidative stress and glycemic regulation," Metabolism: Clinical and Experimental, vol. 49, no. 2, pp. 27-29, 2000.

[38] E. Middleton Jr., C. Kandaswami, and T. C. Theoharides, "The effects of plant flavonoids on mammalian cells: implications for inflammation, heart disease, and cancer," Pharmacological Reviews, vol. 52, no. 4, pp. 673-751, 2000.

[39] R. Rahimi, S. Nikfar, B. Larijani, and M. Abdollahi, “A review on the role of antioxidants in the management of diabetes and its complications," Biomedicine \& Pharmacotherapy, vol. 59, no. 7, pp. 365-373, 2005.

[40] J. S. L. de Munter, F. B. Hu, D. Spiegelman, M. Franz, and R. M. van Dam, "Whole grain, bran, and germ intake and risk of type 2 diabetes: a prospective cohort study and systematic review," PLoS Medicine, vol. 4, no. 8, article e261, 2007.

[41] J. Montonen, P. Knekt, R. Jarvinen, and A. Reunanen, "Dietary antioxidant intake and risk of type 2 diabetes," Diabetes Care, vol. 27, no. 2, pp. 362-366, 2004.

[42] M. Tiedge, S. Lortz, J. Drinkgern, and S. Lenzen, "Relation between antioxidant enzyme gene expression and antioxidative defense status of insulin-producing cells," Diabetes, vol. 46, no. 11, pp. 1733-1742, 1997.

[43] L. Franzini, D. Ardigo, and I. Zavaroni, "Dietary antioxidants and glucose metabolism," Current Opinion in Clinical Nutrition and Metabolic Care, vol. 11, no. 4, pp. 471-476, 2008.

[44] O. Coskun, M. Kanter, A. Korkmaz, and S. Oter, "Quercetin, a flavonoid antioxidant, prevents and protects streptozotocininduced oxidative stress and beta-cell damage in rat pancreas," Pharmacological Research, vol. 51, no. 2, pp. 117-123, 2005.

[45] L. A. Pham-Huy, H. He, and C. Pham-Huy, "Free radicals, antioxidants in disease and health," International journal of Biomedical Science, vol. 4, no. 2, pp. 89-96, 2008. 
[46] M. Georgoulis, E. Fragopoulou, M. D. Kontogianni et al., "Blood redox status is associated with the likelihood of nonalcoholic fatty liver disease irrespectively of diet's total antioxidant capacity," Nutrition Research, vol. 35, no. 1, pp. 41-48, 2015.

[47] Z. Bahadoran, M. Golzarand, P. Mirmiran, N. Shiva, and F. Azizi, "Dietary total antioxidant capacity and the occurrence of metabolic syndrome and its components after a 3-year follow-up in adults: Tehran Lipid and Glucose Study," Nutrition \& Metabolism, vol. 9, no. 1, p. 70, 2012.

[48] F. Saura-Calixto, "Dietary fiber as a carrier of dietary antioxidants: an essential physiological function," Journal of Agricultural and Food Chemistry, vol. 59, no. 1, pp. 43-49, 2011. 